\title{
Design and Optimisation of WDM Circular Photonic Crystals Characterised by Induced Anisotropy
}

\author{
Alessandro Massaro*, Roberto Cingolani, Massimo De Vittorio, and Adriana Passaseo ${ }^{\dagger}$ \\ National Nanotechnology Laboratory of CNR-INFM, Distretto Tecnologico-ISUFI, Università del Salento, \\ Via Arnesano, 73100 Lecce, Italy
}

\begin{abstract}
In this work we analyze the birefringence effect in circular photonic crystals such as dielectric rings (DR) and photonic crystals with air holes arranged in circular patterns. The dielectric concentric circular patterns admit two preferred electric fields orthogonal components defined by an extraordinary and an ordinary refractive index. These electric fields, localized in the central region of the circular photonic crystal (CPC) will be radiated at different wavelengths. This behaviour allows to characterize the analysed structures as wavelength division multiplexers. We first analyze the induced anisotropy of the multiplexers, and, then, we study the wavelength selectivity. Similar emitted wavelengths related to a air-hole CPC and to a corresponding DR structure are observed. The multiplexing behaviour is numerically modelled by the finite element method approach which provides the emitted resonant wavelengths and the quality Q-factors for a membrane-type optical wavelength division multiplexer obtained by the CPC design.
\end{abstract}

Keywords: Anisotropy, Circular Photonic Crystal, Microcavity, Optical Wavelength Division Multiplexer.

\section{INTRODUCTION}

The birefringent properties of crystals may be explained in terms of the anisotropic electrical properties of molecules of which the crystals are composed. Birefringence may, however, arise from anisotropy on a scale much larger than molecular, namely when there is an order arrangement of similar particles of optically isotropic material whose size is large compared with the dimensions of molecules, but small compared with the wavelength of light. We then speak of form birefringence. The form birefringence, ${ }^{1-10}$ which induces artificial anisotropy in the structure, performs the important function of separating an incident beam in two orthogonally polarized outgoing parallel beams. Such a function can be accomplished by isotropic periodic dielectric structures that can split an incident beam in two preferred directions as in Bragg reflectors, polarization splitters, and wavelength division multiplexers (WDM). In particular is very interesting to apply the anisotropic property to dielectric rings (DR) illustrated in Figure 1(a). In fact the circular dielectric patterns, ${ }^{6,10-14}$ arranged in ring configuration, are suitable for practical

\footnotetext{
${ }^{*}$ Author to whom correspondence should be addressed.

${ }^{\dagger}$ Permanent address: IMM-CNR sezione Lecce, University Campus, Lecce-Monteroni 73100, Italy.
}

applications in which whispering gallery modes (WGM) ${ }^{11}$ are requested for high quality factors in large geometrical dimensions. This configuration is also suitable for devices in which the localized bound states, supported by cavity defects, generate different wavelength emissions associated to modes correlated to the polarization. ${ }^{6,15}$ The presence of main modes associated to different electric field polarizations can be explained by the form birefringence effect. From optical theory ${ }^{2,5}$ the form birefringence is explained by considering the idealized case of a regular assembly of particles that have the form of thin parallel plates. This idealized case can be extended to dielectric multilayered structures with circular geometries as DR and CPC devices. Previous works have analyzed the Bragg confinement or reflection ${ }^{6,16}$ by studying ring resonators and CPC devices with micro-cavity or guiding defect. According to momentum conservation law, the two modes with different polarizations are generated through a Bragg grating arranged in a circular patterns. ${ }^{5,6,8,9,16}$ Starting from the anisotropy induced by a dielectric multilayer stack, ${ }^{2,5}$ the Bragg theory and the birefringence effect can be also applied to DR and CPC structures by considering the following considerations: by rotating the thin film parallel plates of Figure 3(a) it is possible to apply the Bragg theory to a DR device where each ring 
(a)

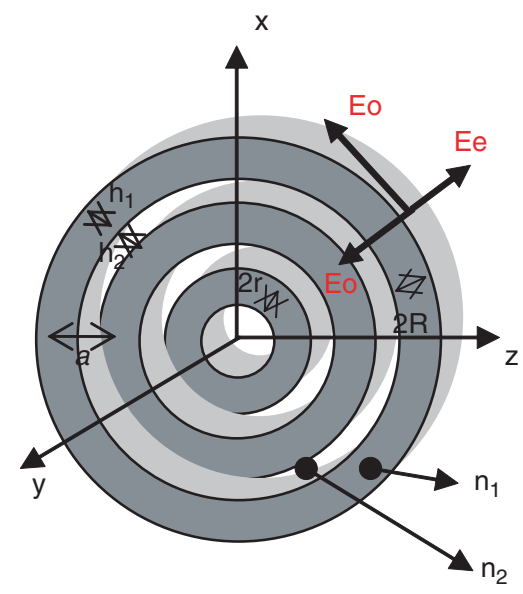

(b)

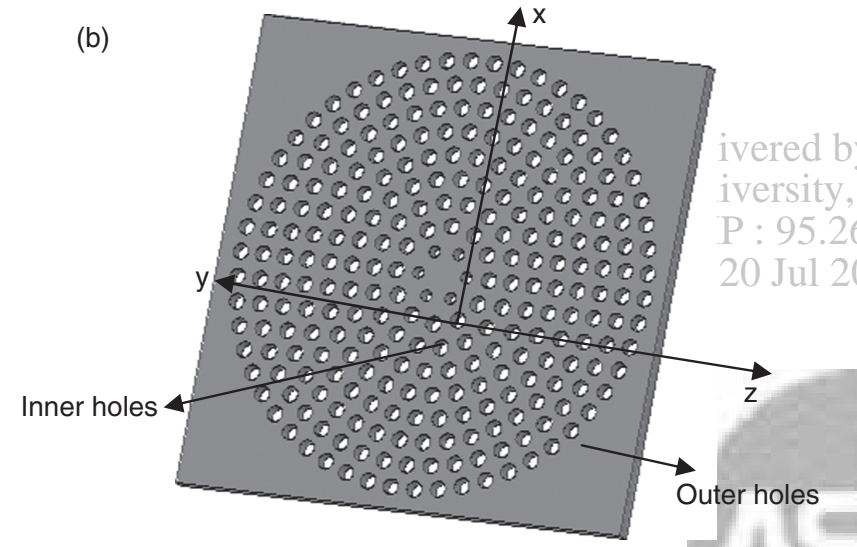

Fig. 1. (a) Concentric dielectric air rings $\left(n_{1}=1\right)$ in GaAs material $\left(n_{2}=3.408\right)$. (b) CPC with cylindrical air holes. The six inner holes define the central micro-cavity.

is the geometrical rotation of a single layer as shown in the scheme of Figure 3(b), moreover, an air ring is the approximation of infinite air cylinders arranged in a circular pattern (see Fig. 3(c), therefore, all the considerations about the Bragg and birefringence effects are suitable also for a CPC. By considering appropriate physical conditions and combinations of materials and dimensions, the DR and the CPC structures behave as WDM, where two resonant modes associated with the polarization (ordinary mode and extraordinary mode) are radiated in the vertical $y$-direction. The two modes are not localized in a bandgap region, and, are associated to an ordinary $\mathbf{E}_{\mathrm{o}}$ electric field, and to an extraordinary $\mathbf{E}_{\mathrm{e}}$ electric field. As shown in Figure 1(a), $\mathbf{E}_{\mathrm{o}}$ is defined tangential to the rings and to the air holes, and $\mathbf{E}_{\mathrm{e}}$ is defined radial to the rings. The ordinary electric field component is characterized by an effective ordinary refractive index $n_{\mathrm{o}}$, and, the extraordinary electric field component is characterized by an effective extraordinary refractive index $n_{\mathrm{e}}$ which changes with the incidence angle of the source. Both the refractive indices define the modes of the analyzed WDM. We have analyzed in a previous work ${ }^{16}$ the analogy of the birefringent behaviours of

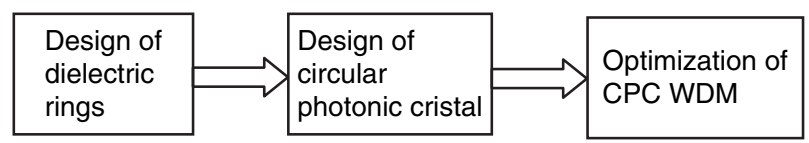

Fig. 2. Block diagram: design and optimisation of the WDM CPC.

DR and CPC structures, without considering the effects of geometrical parameters on the frequency responses. In this work we study how it is possible to separate two emitted wavelengths generated by the induced anisotropy, simply by changing the ring thicknesses or equivalently the air holes diameters. Moreover for the first time we propose the evolution of the design and the optimization of a circular WDM with CPC configuration: the design starts from a DR basic structure and ends with an optimized membrane type CPC WDM. We summarize the proposed work in the points illustrated in the block diagram of Figure 2. In particular:

(i) We first: design division wavelength multiplexers with dielectric rings: the design provides two main modes (WGM modes) in a range between $1 \mu \mathrm{m}$ and $1.40 \mu \mathrm{m}$ : the two main modes (associated to an ordinary and an extraordinary electric field polarization) are calculated by varying the ring thicknesses.

(ii) In order to obtain high quality factors we design the corresponding CPC (the CPC is suited for membrane type configuration characterized by high quality factors ${ }^{17}$ ): we prove the equivalence between the DR and the CPC configuration through the evaluation of the extraordinary refractive indices of both the structures, and through the transmittivity responses calculated by a finite element method (FEM) tool. ${ }^{17}$

(iii) We optimize the equivalent CPC WDM by shifting the inner air holes and by considering a properly designed membrane type configuration.

\section{THEORY: WAVE PROPAGATION IN CIRCULAR PHOTONIC CRYSTALS}

As in negative uniaxial crystals, ${ }^{5}$ dielectric periodic multilayer structures select two modes (the ordinary and extraordinary one) which propagate in the structure. These two modes are characterized by an extraordinay $\mathbf{E}_{\mathrm{e}}$ field component (orthogonal to the ring) and by an ordinary $\mathbf{E}_{\mathrm{o}}$ component tangential to the ring. These two modes are characterized by a split angle $\phi$ (split angle related to the ordinary and extraordinary field components in the periodic $x-z$ plane) estimated by means of Huygens principle: starting from the ordinary and extraordinary wave fronts $\mathrm{s}^{2,3,5}$ the ordinary and the extraordinary wave fronts can be superimposed to the indices ellipse for particular $\tau$ values ( $\tau$ is the propagation time of the wave front in the periodic structure). According to Figure 4(a), for plane wave incidence $\Sigma^{\prime}$ is the envelope, at the time $t+\tau$, of 


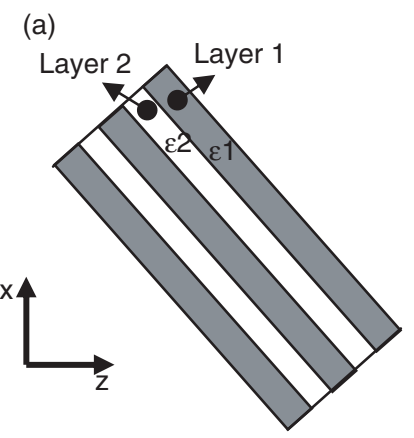

(b)

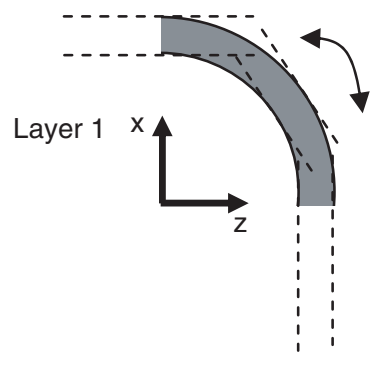

(c)

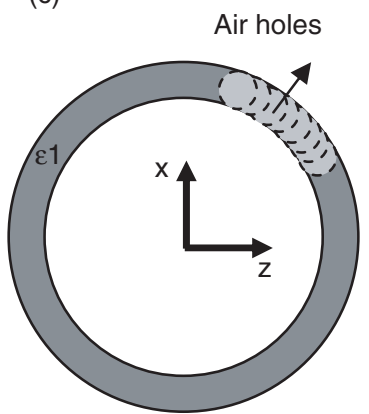

Fig. 3. (a) Anisotropic dielectric multilayer stack. ${ }^{5}$ (b) Geometrical construction of dielectric ring obtained as rotation of a single layer. (c) Air ring approximation $\left(\varepsilon_{x}=1\right)$ : the air ring approximation corresponds to infinite air cylinders arranged in a circular pattern.

wavelets emitted from the various points of the wave front $\Sigma$ at the time $t$. We see from Figure 4(a) that $\mathrm{ON} / \tau$ is the velocity and $\mathrm{OQ} / \tau$ is the propagation velocity of the wave. By taking into account the reference system of Figure 4(a) and a wavelet centered in $\mathrm{O}$, we have $\chi=\pi / 2-\chi^{\prime}, d \chi^{\prime}=$ $-d \chi, x=-n \cdot \cos \chi^{\prime}, z=-n \cdot \sin \chi^{\prime}$, where $n=n_{\mathrm{e}}\left(\chi^{\prime}\right)=$ $\mathrm{OB}=\mathrm{OA}$ is the refractive index of the $\Sigma$ envelope: The ellipse equation in the $x-z$ plane for an optical axis orientation $\chi^{\prime}$ is:

$$
\frac{\cos ^{2} \chi^{\prime}}{n_{\mathrm{e}}^{2}}+\frac{\sin ^{2} \chi^{\prime}}{n_{\mathrm{o}}^{2}}=\frac{1}{n^{2}}
$$

By differentiation we get $^{3,5}$

$$
\frac{d n}{d \chi^{\prime}}=n^{3} \cdot\left(\frac{1}{n_{\mathrm{e}}^{2}}-\frac{1}{n_{\mathrm{o}}^{2}}\right) \cdot \sin \chi^{\prime} \cdot \cos \chi^{\prime}
$$

The relation between the $\phi$ angle and the variation of the refractive index $n$ with respect to the angle $\chi^{\prime}$ is as follows: ${ }^{3,5}$

$$
\tan \phi=\frac{1}{n} \cdot \frac{d n}{d \chi^{\prime}}=-n^{2} \cdot\left(\frac{1}{n_{\mathrm{e}}^{2}}-\frac{1}{n_{\mathrm{o}}^{2}}\right) \cdot \sin \chi^{\prime} \cdot \cos \chi^{\prime}
$$

where $36: 10$

$$
n^{2}=\frac{n_{\mathrm{e}}^{2} \cdot n_{\mathrm{o}}^{2}}{n_{\mathrm{o}}^{2} \cdot \sin ^{2} \chi+n_{\mathrm{e}}^{2} \cdot \cos ^{2} \chi}
$$

with the ordinary and the extraordinary refractive indices defined as

$$
\begin{aligned}
& n_{\mathrm{e}}^{2}=\frac{n_{1}^{2} n_{2}^{2}}{f_{1} n_{2}^{2}+f_{2} \varepsilon_{1}} \\
& n_{\mathrm{o}}^{2}=f_{1} n_{1}^{2}+f_{2} n_{2}^{2}
\end{aligned}
$$

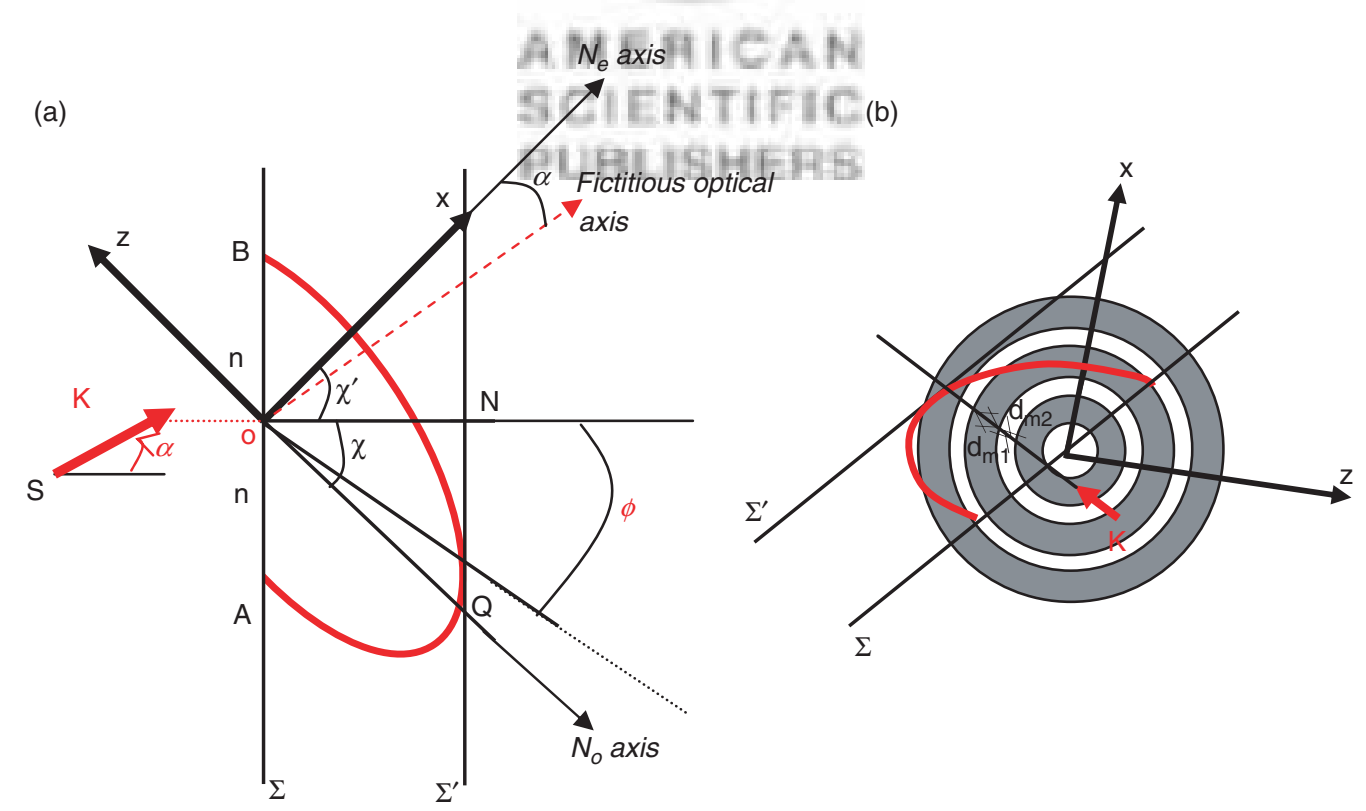

Fig. 4. (a) Wave front and refractive index ellipse related to the DR device at a particular propagation time; the value $n=n_{\mathrm{e}}\left(\chi^{\prime}\right)=\mathrm{OB}$ changes with the $\mathbf{K}$ direction. The direction of $\mathbf{K}$ is in the $x-z$-plane of the periodic structure. (b) Superposition of the ellipse defined in Figure 2(b) with the DR. 
with

$$
\begin{aligned}
& f_{1}=\frac{\sum_{n} d_{n 1}}{\sum_{n} d_{n 1}+\sum_{m} d_{m 2}} \\
& f_{2}=\frac{\sum_{n} d_{n 2}}{\sum_{n} d_{n 1}+\sum_{m} d_{m 2}}
\end{aligned}
$$

We observe that the filling factors $f_{1}$ and $f_{2}$ of the ring structure are obtained by $d_{n 1}$ and $d_{m 2}$ which are the segments defined by the propagation direction of the $\mathbf{K}$ vector (see Fig. 4(b)). A properly designed circular geometry will provide the confinement of the two modes in a central region: by choosing particular geometrical dimensions such as $h_{1}$ and $h_{2}$, the two modes, generated by the induced anisotropic effect and initially separated by a split angle, will converge to the central region. This central field confinement will represent the resonances of the artificial anisotropic structures. In this way the devices of Figure 1(a) and (b) will behave as micro-cavity resonators ${ }^{17}$ and the ordinary and the extraordinary modes will be radiated along the $y$-vertical direction as in multiplexing applications. Due to the ring approximation reported in Figure $3(\mathrm{c})$, the behavior of the CPC with cylindrical air holes is similar to the corresponding DR structure: in fact, the $\mathrm{DR}$ is the extended case of infinite air holes arranged in a circular pattern. The analogy is proved in Figure 5 by comparing the extraordinary refractive index of the CPC (numerical results obtained by a FORTRAN code which calculates the filling factors of Eqs. (5) and (6)) with the analytical extraordinary refractive index related to the corresponding DR (obtained through the equivalence with the thin film parallel plates equivalent structure. ${ }^{6}$ ) The corresponding structures of Figure 1(a) and (b) are obtained by

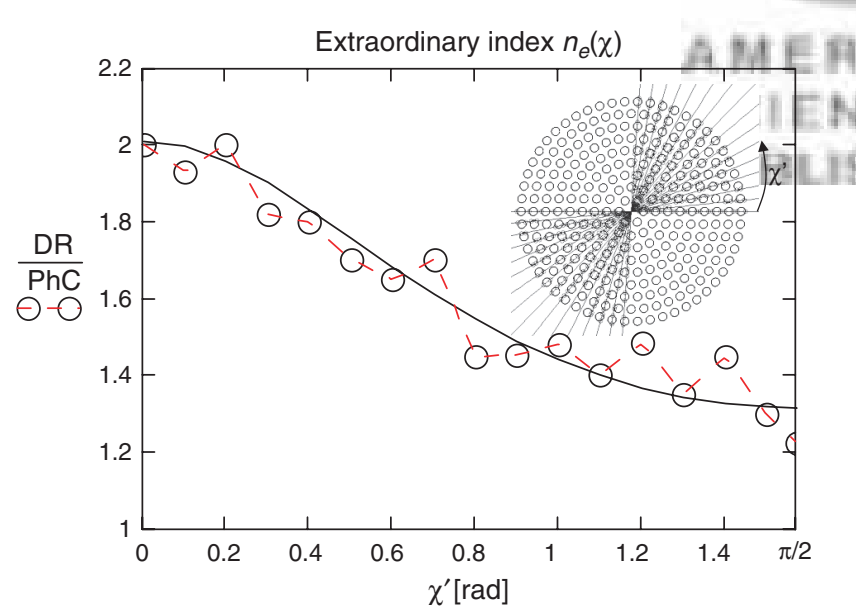

Fig. 5. Extraordinary refractive index $n_{\mathrm{e}}\left(\chi^{\prime}\right)$ versus the optical axis orientation $\chi^{\prime}$. The solid line refers to the DR structure of Figure 1(a) with $h_{1}=2 R=240 \mathrm{~nm}, h_{2}=a-h_{1}$, and lattice constant $a=350 \mathrm{~nm}$. The numerical (FORTRAN code implementation) results are obtained for the equivalent ring structure through the possible combinations of incidence angles which define the orientation $\chi^{\prime}$. Inset: schematic diagram of the numerical FORTRAN approach which defines the filling factors $f_{1}$ and $f_{2}$ and, consecutively, the extraordinary refractive index of Eq. (4). The numerical results refers to the CPC of Figure 1(b).

considering the air ring thicknesses equals to the cylindrical air hole diameters.

\section{FEM DESIGN AND MODELLING}

The GaAs CPC of Figure 1(b) is an unconventional kind of quasi-3D photonic crystal with circular lattice pattern: it consists of air holes in a GaAs material $\left(n_{2}=3.408\right)$ along circular concentric lines. This particular circular geometry has peculiar behaviour if compared with the traditional square and triangular lattices, but it is difficult to model by using conventional numerical approaches such as wave expansion method. If the cylindrical air holes are properly designed, the degenerate modes, obtained by the induced anisotropy and generated in the circular structure, can be confined in a central micro-cavity region by increasing the power radiated above the cavity. A proper tuning of the inner holes of Figure 1(b) (holes shift and diameters variation) can provide an high intensity microcavity resonance. For this purpose the inner holes have a reduced radius and are shifted along the radial outward direction, in order to improve the resonance properties. In particular the designed GaAs CPC is characterized by lattice constant $a=350 \mathrm{~nm}$, outer holes radius of $R=120-125 \mathrm{~nm}$, inner hole radius $r=70 \mathrm{~nm}$, and slab thickness of $200 \mathrm{~nm}$. We analyze the microcavity resonances of the degenerate mode by a three-dimensional 3D FEM tool ${ }^{6}$ typically used to design CPC. The modeling is based on the definition of

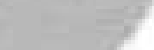


the unit cell ${ }^{16}$ as a 3D CPC. The CPC unit cell is characterized by resonance field, radiation field and by scattering parameters. In the 3D FEM modelling, the interface between all the $3 \mathrm{D}$ objects and the background is a perfect 'absorbing boundary' through which no energy may enter or exit. Wave ports are typically placed at this interface in order to provide a window that couples the model device to the external world. In our case we set the ports as illustrated in Figure 6, where the input port I.P. is placed on the excitation side, and the output port O.P. is placed above the central cavity region. The transmittivity response represents the electric field transmission between the input and output port. In order to distinguish the ordinary mode and the extraordinary one we excite from the input port with different electric field polarizations: the $\mathbf{E}_{\mathrm{o}}$ excitation, shown in Figure 6, will select the transmittivity of the ordinary mode, while, the $\mathbf{E}_{\mathrm{e}}$ excitation (also indicated in Fig. 6) will characterize the transmittivity of the extraordinary mode. This field configuration is in agreement with the ordinary and extraordinary field configuration of an anisotropic structure. We observe that, according with the polarization of the source and with the plane-wave definition, the $\mathbf{E}_{\mathrm{o}}$ excitation correspond to a transverse electric (TE) source, and the $\mathbf{E}_{\mathrm{e}}$ correspond to a transverse magnetic (TM) source.

The $Q$ factor (FEM solution) is evaluated as ${ }^{17}$

$$
Q=2 \pi f \frac{U}{P}
$$

where $U$ is the energy stored inside the CPC, $P$ is the power lost through a domain which encloses the whole structure, and $f$ is the working frequency.

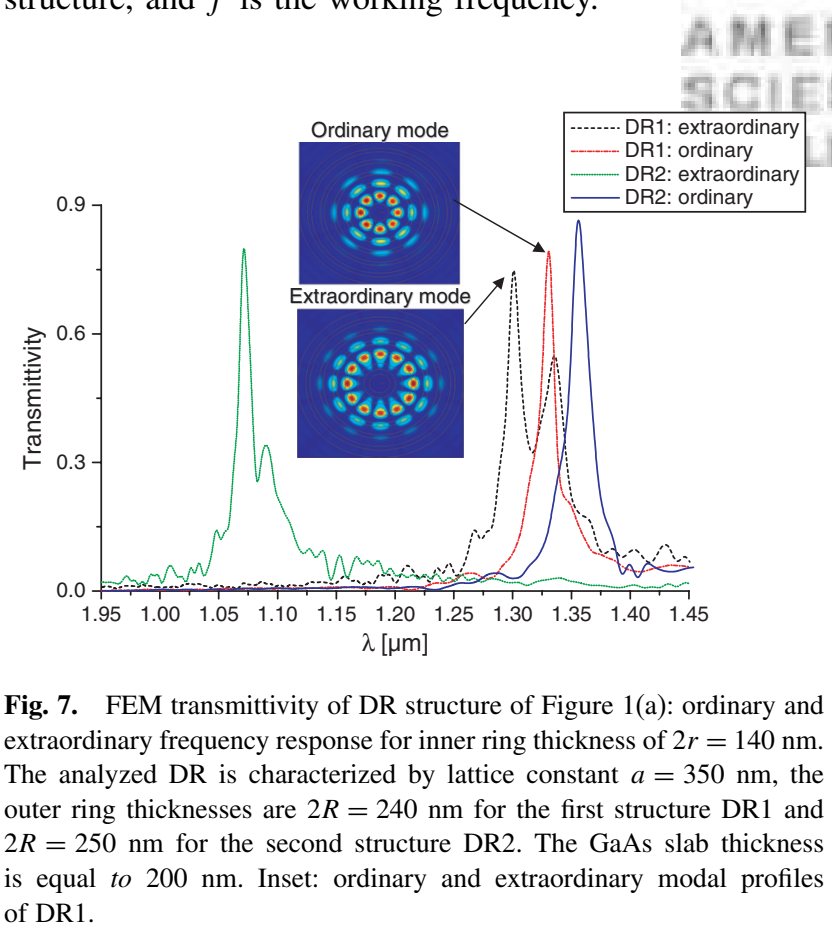

Fig. 7. FEM transmittivity of DR structure of Figure 1(a): ordinary and extraordinary frequency response for inner ring thickness of $2 r=140 \mathrm{~nm}$. The analyzed DR is characterized by lattice constant $a=350 \mathrm{~nm}$, the outer ring thicknesses are $2 R=240 \mathrm{~nm}$ for the first structure DR1 and $2 R=250 \mathrm{~nm}$ for the second structure DR2. The GaAs slab thickness is equal to $200 \mathrm{~nm}$. Inset: ordinary and extraordinary modal profiles of DR1.

\section{RESULTS AND DISCUSSION}

In Figures 7 and 8 are reported the ordinary and the extraordinary frequency responses by changing the outer holes diameter of the CPC, and the ring thicknesses of the corresponding DR. In particular Figures 7 and 8 consider a ring thickness of $240 \mathrm{~nm}$ and $250 \mathrm{~nm}$, and air holes diameters $2 R=240 \mathrm{~nm}$, and $2 R=250 \mathrm{~nm}$ for the corresponding CPC. In both the structures we observe wavelength peaks associated to the ordinary/extraordinary polarised fields, but the devices with ring thickness or air holes diameter of $250 \mathrm{~nm}$ show a better wavelength division with well distinguished wavelength peaks. Moreover we found a WGM profile for the DR structure and a microcavity modes confinement for the corresponding CPC (see insets of Figs. 7 and 8). These peaks are also reported in Table I which shows the similar emitted wavelengths

Fig. 8. FEM transmittivity of CPC structure of Figure 1(b): ordinary and extraordinary frequency response for inner holes radius of $r=70 \mathrm{~nm}$. The analysed CPC is characterized by lattice constant $a=350 \mathrm{~nm}$, the outer holes radius are $R=240 \mathrm{~nm}$ for the first structure PhC1 and $R=$ $250 \mathrm{~nm}$ for the second structure CPC2. The GaAs slab thickness is equal to $200 \mathrm{~nm}$. Inset: ordinary and extraordinary modal profiles of CPC1.

Table I. Emitted wavelengths of the WDM related to Figures 7, 8 and 9.

\begin{tabular}{lllllc}
\hline & DR1 & CPC1 & DR2 & CPC2 & CPC2 opt. \\
\hline$\lambda_{\text {ordinary }}[\mu \mathrm{m}]$ & 1.33 & 1.32 & 1.36 & 1.335 & 1.363 \\
$\lambda_{\text {extraordinary }}[\mu \mathrm{m}]$ & 1.365 & 1.36 & 1.075 & 1.12 & 1.079 \\
\hline
\end{tabular}

Table II. Errors of emitted wavelengths $\Delta \lambda$ and maxima quality factors $\mathrm{Q}$ of $\mathrm{CPC} 1, \mathrm{CPC} 2$ and optimised CPC2.

\begin{tabular}{lcccc}
\hline & $\Delta \lambda_{\text {ordinary }}[\mu \mathrm{m}]$ & $\Delta \lambda_{\text {extraordinary }}[\mu \mathrm{m}]$ & $\mathbf{Q}_{\text {ordinary }}$ & $\mathbf{Q}_{\text {extraordinary }}$ \\
\hline $\mathrm{CPC} 1$ & 0.01 & 0.005 & $\sim 61000$ & $\sim 30000$ \\
$\mathrm{CPC} 2$ & 0.005 & 0.045 & $\sim 55000$ & $\sim 25000$ \\
$\mathrm{CPC} 2$ opt. & 0.03 & 0.004 & $\sim 70000$ & $\sim 41000$ \\
\hline
\end{tabular}




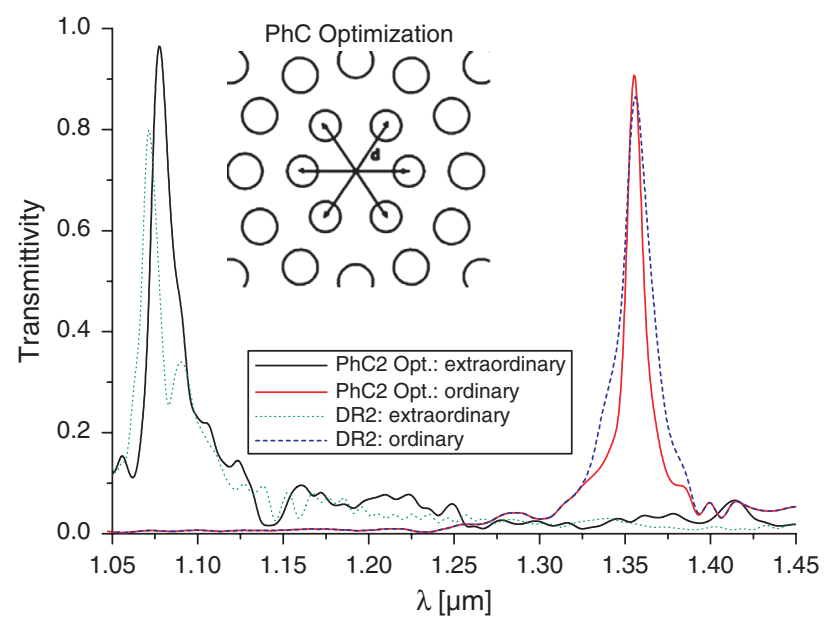

Fig. 9. FEM transmittivity comparison between the DR2 and the CPC2 optimised (CPC2 Opt.) structures. $d=370 \mathrm{~nm}$ represent the inner holes shift.

Delivered b of both the structures, by proving that the DR design provides approximately the same frequency behaviour of the CPC structure, and, therefore, represents a first step of the design process. The low difference of the peaks positions between the DR and the CPC is due to the ring geometrical approximation: the limits of the ring approximation is also represented by the oscillating behaviour of the effective refractive index of Figure 5.

So, assuming as holes diameter the value of the ring thickness, both the structures are characterised by similar emitted wavelengths: DR1 and CPC1 are characterised by similar emitted $\lambda$ values (see Table I) but does not present a good selectivity between the ordinary and the extraordinary wavelength, whereas, DR2 and CPC 2 present distinguished frequency bands (see Figs. 7 and 8). The results reported in the Table II prove the design equivalence of both the structures: in fact, low errors $\Delta \lambda_{\text {ordinary, extraordinary }}(\mathrm{CPC} 1)=\mid \lambda_{\text {ordinary, extraordinary }}(\mathrm{CPC} 1)-$ $\lambda_{\text {ordinary, extraordinary }}(\mathrm{DR} 1) \mid$ and $\Delta \lambda_{\text {ordinary, extraordinary }}(\mathrm{CPC} 2)=$ $\left|\lambda_{\text {ordinary, extraordinary }}(\mathrm{CPC} 2)-\lambda_{\text {ordinary, extraordinary }}(\mathrm{DR} 2)\right|$ related to the peak positions are found. Moreover, concerning the WDM CPC2, it is possible to decrease the error $\Delta \lambda_{\text {ordinary, extraordinary }}(\mathrm{CPC} 2)$ by considering an optimized CPC2 (CPC2 Opt.): as shown in Figure 9, the wavelengths peaks of CPC2 Opt. coincide with the DR2 one. The optimised CPC2 opt. devices is obtained by considering a distance of the inner holes from the central axis of the cavity of $d=370 \mathrm{~nm}$ (see inset of Fig. 9).

An high radiated power of the designed CPC can be obtained by considering a membrane configuration. In this case the air cladding thickness below the CPC slab enhances the radiated power in the region above the microcavity. ${ }^{17}$ We estimate this enhancement through the quality $Q$-factor. The air cladding layer behaves as an air cavity resonator that, if properly sized (nominal depth as $3 \lambda_{0} / 2$ or odd multiples of $3 \lambda_{0} / 2$ ), should provide electromagnetic field enhancement of the GaAs waveguide layer. By varying the air cavity depth from the nominal value in steps of $20 \mathrm{~nm}$, the $Q$ factor drastically decreases (as shown in Fig. 10). In particular, we observe from Figure 10 that the optimized CPC2 presents $Q$ values higher than the other membrane type CPC: we found numerically that for an air cavity depth of $3 \lambda_{0} / 2$ a maximum $Q$ factor of the order of $7 * 10^{4}$ for the ordinary mode and a maximum $Q$ factor of the order of $4 * 10^{4}$ for the extraordinary mode.

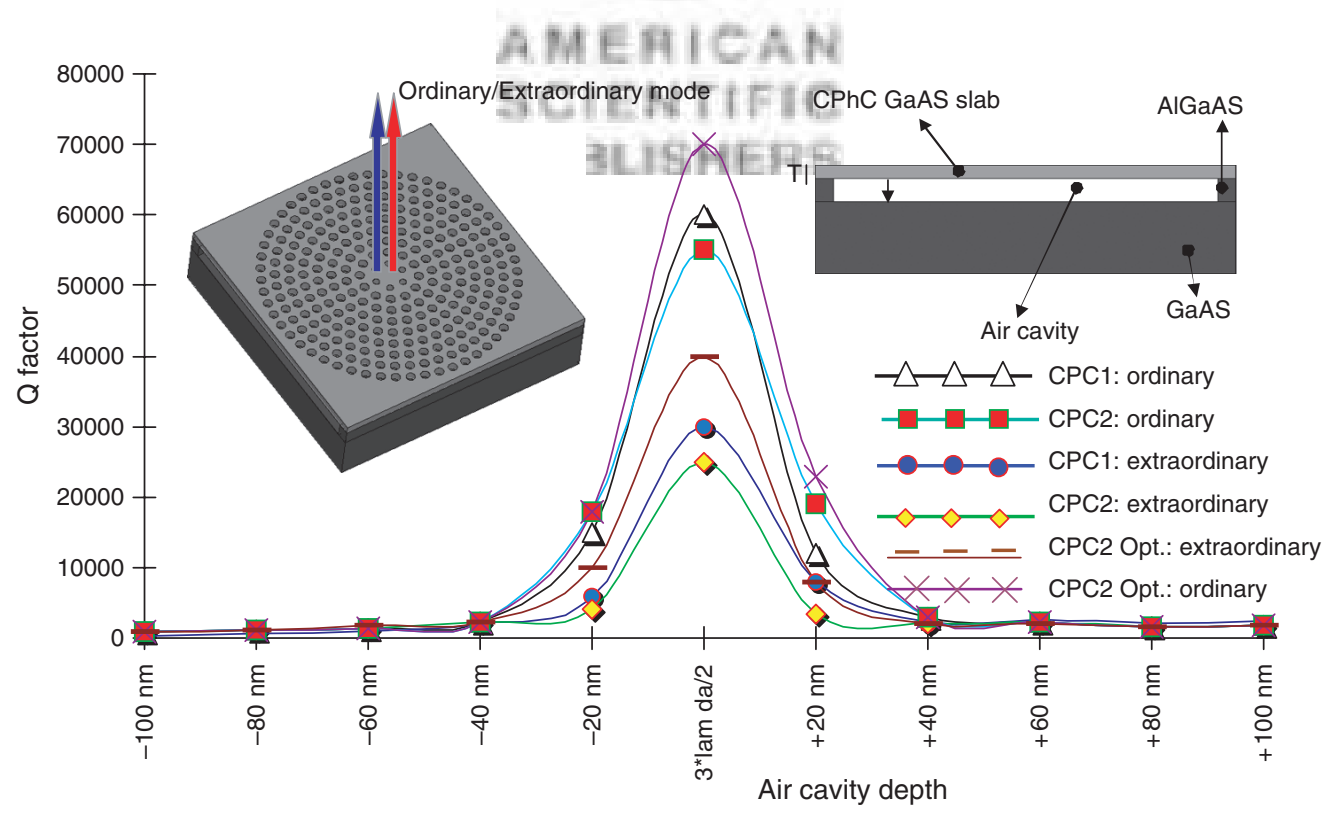

Fig. 10. Ordinary and extraordinary Q factor FEM evaluation as a function of the air cladding thickness below the CPC slabs of Figures 7, 8, and 9. Inset right: cross section of membrane type CPC with slab thickness $T=200 \mathrm{~nm}$. Inset left: schematic plot of the radiated power direction related to the WDM device. 


\section{CONCLUSION}

In this work, two unconventional kind of WDM based on a circular geometry were presented. Both the structures select two wavelengths generated by the induced anisotropy. The presented model highlights the analogy between the DR and CPC configurations. This analogy is also proved through the comparison of the effective refractive indices. Finally, the model provides the optimization of a membrane type CPC.

\section{References}

1. F. Abelès, Ann. de Physique 5, 596 (1950).

2. M. Born and E. Wolf, Principles of Optics, 6th edn., Cambridge University Press, Cambridge, UK (1980), Chap. 14.

3. B. Rossi, Optics, Addison-Wesley, Milano, Italy (1967), Chap. 6.

4. K. Shiraishi and K. Matsumura, IEEE J. Quantum Electron. 30, 2417 (1994).

5. A. Massaro, L. Pierantoni, and T. Rozzi, IEEE J. Quantum Electron. $\underline{40,821} \underline{\text { (2004)}}$.
6. A. Massaro, V. Errico, R. Cingolani, M. Devittorio, and A. Passaseo, IEEE J. Quantum Electron. 44, 1225 (2008).

7. A. D. Remenyuk, E. V. Astrova, T. S. Perova, V. A. Tolmachev, and J. A. Moore, IEEE Proceeding ICTON 2003 1, 269 (2003).

8. P. Yeh, Introduction to Photorefractive Nonlinear Optics, WileyInterscience, New York (1993), Chap. 2.

9. P. Yeh, Optical Waves in Layered Media, Wiley, New York (1988).

10. A. Yariv, Optical Waves in Crystals, Wiley, New York (1984).

11. P. T. Lee, T. W. Lu, and F. M. Tsai, IEEE Photon. Technol. Lett. 19,710 (2007).

12. P. T. Lee, T. W. Lu, J. H. Fan, and F. M. Tsai, Appl. Phys. Lett. 90, 151125 $\underline{\text { (2007) }}$.

13. J. Scheuer and A. Yariv, IEEE J. Quantum Electron. 39, 1555 (2003).

14. J. Scheuer and A. Yariv, Phys. Rev. E 70, 036603 (2004).

15. A. Tandaechanurat, S. Iwamoto, M. Nomura, N. Kumagai, and Y. Arakawa, Opt. Express 16, 448 (2008).

16. J. Scheuer and A. Yariv, Opt. Express 11, 21 (2003).

17. A. Massaro, V. Errico, T. Stomeo, A. Salhi, R. Cingolani, A. Passaseo, and M. De Vittorio, IEEE Journal of Light. Technol. 26,2960 (2008).

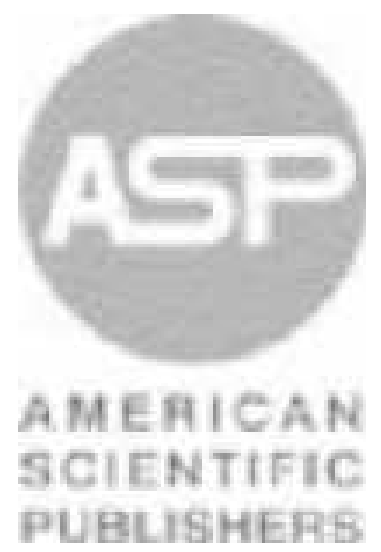

